\title{
HAEMOCOMPATIBILITY EVALUATION OF DLC- AND SIC-COATED SURFACES
}

\author{
N. Nurdin ${ }^{1 *}$, P. François ${ }^{2}$, Y. Mugnier ${ }^{1}$, J. Krumeich ${ }^{3}$, M. Moret ${ }^{1}$, B.-O. Aronsson ${ }^{1}$ and P. Descouts ${ }^{1}$ \\ ${ }^{1}$ Group of Applied Biomedical Physics, University of Geneva, CH-1211 Geneva, \\ ${ }^{2}$ Division of Infectious Diseases, University Hospital of Geneva, CH-1211 Geneva \\ ${ }^{3}$ Sulzer Innotec, Materials and Surface Engineering 1512, CH-8404 Winterthur, Switzerland
}

\begin{abstract}
Diamond-like carbon (DLC) and silicon carbide (SiC) coatings are attractive because of low friction coefficient, high hardness, chemical inertness and smooth finish, which they provide to biomedical devices. Silicon wafers $\left(\mathrm{Si}_{\text {waf }}\right)$ and silicone rubber $\left(\mathrm{Si}_{\text {rub }}\right)$ plates were coated using plasmaenhanced chemical vapour deposition (PE-CVD) techniques. This article describes: 1- the characterization of modified surfaces using attenuated total reflection-Fourier transform infrared spectroscopy (ATR/FTIR) and contact angle measurements, 2- the results of three in-vitro haemocompatibility assays. Coated surfaces were compared to uncoated materials and various substrates such as polymethylmethacrylate (PMMA), polyethylene (LDPE), polydimethylsiloxane (PDMS) and medical steel (MS). Thrombin generation, blood platelet adhesion and complement convertase activity tests revealed the following classification, from the most to the least heamocompatible surface: $\mathrm{Si}_{\text {rub }} / \mathrm{DLC}-\mathrm{Si}_{\text {rub }} / \mathrm{DLC}-\mathrm{Si}_{\text {wat }} / \mathrm{LDPE} / \mathrm{PDMS} / \mathrm{SiC}-$ $\mathrm{Si}_{\text {wat }} / \mathrm{Si}_{\text {wat }} /$ PMMA/ MS. The DLC coating surfaces delayed the clotting time, tended to inhibit the platelet and complement convertase activation, whereas $\mathrm{SiC}$-coated silicon wafer can be considered as thrombogenic. This study has taken into account three events of the blood activation: coagulation, platelet activation and inflammation. The response to those events is an indicator of the in vitro haemocompatibility of the different surfaces and it allows us to select biomaterials for further in vivo blood contacting investigations.
\end{abstract}

Key words: Diamond-like carbon coating, plasma deposition, platelet adhesion, thrombin generation, complement convertase attachment.

*Address for correspondence:

N. Nurdin

Group of Applied Biomedical Physics,

University of Geneva,

20 rue de l'Ecole de Médecine,

1211 Geneva 4, Switzerland

FAX Number: 41(0) 227810980

E-mail: nathalie.nurdin@physics.unige.ch

\section{Introduction}

The purpose of this study was to evaluate in vitro the haemocompatibility of hydrogenated diamond-like carbon (DLC) and silicon carbide ( $\mathrm{SiC}$ ) coatings deposited by plasma-enhanced chemical vapour deposition (PECVD) on silicon wafers $\left(\mathrm{Si}_{\text {waf }}\right)$ and silicon rubber plates $\left(\mathrm{Si}_{\text {rub }}\right)$ and to compare their in vitro biological performances to conventional biomaterials. DLC coatings are known for their high values of hardness, low friction coefficients, chemical inertness and smooth surface finish (Jones et al., 2000). Thus, they allow the reduction of metal ion release by avoiding formation of wear fragments, and they show a relatively good biocompatibility with no significant levels of cellular toxicity (Jones $e t$ al., 1999). They can be employed to modify the surface of different implants, e.g. metallic intracoronary stents whose applications are limited by thrombotic occlusions and restenosis (Gutensohn et al., 2000), bioprosthetic heart valves (Wang et al., 2002) and hip joints where the presence of wear particles can provoke the loosening of the orthopaedic implant (Linder et al., 2002). De Scheerder et al. (2000) have evaluated the biocompatibility of DLC coating in a porcine coronary stent model in vivo. Although thrombosis phenomena are reduced, DLC coating on the stents resulted in an increased inflammatory reaction.

Biomaterials implanted for long periods of time in a human body cannot be considered as totally inert and must meet different criteria to minimize the risks of adverse events. The first major event when a medical device comes in contact with blood is the adsorption of plasma proteins, such as fibrinogen, fibronectin, vitronectin or von Willebrandt factor, as well as tissue and complement factors (Young et al., 1982; Lamba and Cooper, 2001). This adsorption leads to the activation of the blood coagulation cascade and of inflammatory processes that constitute adverse events impairing the implant lifetime. Platelet adhesion followed by aggregation and spreading are the processes by which platelets form thrombus. In the case of a vessel injury, this leads to the formation of a haemostatic plug (George, 2000). The activation is essentially due to thrombin and potent activators secreted by platelets themselves. Thrombin is a proteolytic enzyme that promotes the activity of several clotting factors and acts in the final common pathway of the blood clotting process inducing the cleavage of fibrinogen to fibrin (Ferry and Morrison, 1947; Dawie and Ratnoff, 1964; MacFarlane 1964; Overduin and de Beer, 2000).

Complement, a series of serum proteins involved in 
the mediation of immune reactions, is known to be activated with implanted or external devices that encounter human blood (such as perfusion devices, columns for externally treating blood, indwelling artificial vascular grafts and vascular shunts). Although complement activation is an important defence mechanism of the host, particularly against microbial infections, complement activation by material components of blood-contacting devices may be harmful to the host and/or affect the function of the device. Complement activation is a tightly regulated process which in addition to direct cell lysis can have profound affects on the immune, vascular and coagulation systems. Inappropriate complement activation by bloodcontacting medical devices may lead to systemic inflammatory reaction directed against the foreign device. The complement activation results in the generation of complexes leading to the production of $\mathrm{C} 3$ and $\mathrm{C} 5$ convertases. These convertases cleave $\mathrm{C} 3$ and $\mathrm{C} 5$ to $\mathrm{C} 3 \mathrm{a}, \mathrm{C} 3 \mathrm{~b}$ and $\mathrm{C} 5 \mathrm{a}$ and $\mathrm{C} 5 \mathrm{~b}$, respectively. These complement factors lead to the synthesis of ligands for the complement receptors on leukocytes and contribute to the initiation of inflammatory processes whose intensity is dependent on the haemocompatibility of the material (Craddock et al., 1977; Bohler et al., 1983; Chenoweth, 1984, 1986).

In this study, human blood was used for the in vitro assessment of haemocompatibility based on the observation of platelet adhesion and activation, thrombin generation and complement convertase production induced by the sample surface.

\section{Materials and Methods}

\begin{abstract}
Materials
Hydrogenated DLC (a-C:H) and SiC films were fabricated on $\mathrm{Si}_{\text {waf }}$ and $\mathrm{Si}_{\text {rub }}$ using the magnetron sputtering techniques. The sputtering facility used was Sulzer Innotec's (Wintherthur, Switzerland) UDP 450 with closed loop unbalanced magnetrons, manufactured by TEER Coatings Ltd., Hartlebury, England. The DLC coatings were prepared using a graphite target and acetylene as reactive gas. The parameters were set to achieve a good coating adhesion and to maintain a low substrate temperature during the deposition process. $\mathrm{SiC}$ was deposited with a silicon target instead of the graphite target used for DLC. Both sides of the samples were coated. The films have a typical thickness of $1 \mu \mathrm{m}$. Medical steel (MS), polydimethylsiloxane (PDMS) and low-density polyethylene (LDPE) plates were provided by HaemoProbe bv (Groningen, The Netherlands) and polymethylmethacrylate (PMMA) plates by the University Hospital of Geneva (Switzerland). The high performance silicone rubber HP-100 was provided by Sulzer Medica after different mechanical and curing treatment $\left(\mathrm{Si}_{\text {rub }}\right)$. All samples were cleaned in 1\% Deconex 11 Universal (Borer Chemie AG, Zuchwil, Switzerland) and subsequently washed with ultra-pure water before experiments.
\end{abstract}

Characterization of sample surface Attenuated total reflection-Fourier transform infrared spectroscopy (ATR-FTIR)

ATR-FTIR analysis was performed on a Perkin-Elmer Paragon 1000 (Hünenberg, Switzerland) equipped with the Golden Gate Single Reflection Diamond ATR (Graceby Inc., Smyrna, GA, USA). Spectra were recorded on an average of 256 scans at a resolution of $4 \mathrm{~cm}^{-1}$.

\section{Static water contact angle measurements}

Wettability of the sample surface was evaluated by measuring the static contact angle between a $2 \mu \mathrm{l}$ deionized water drop and the surface.

\section{Thrombin generation}

The thrombin generation assay was provided by HaemoProbe bv as a ready-to-use test kit (lot $\mathrm{N}^{\circ}$ 020301TGA). This test has been recommended by van Oeveren et al. (2002) for investigations of the coagulation inducing properties of medical devices. Briefly, it can be described as an enzymatic colorimetric assay based on a prepared plasma product that enables the determination of thrombin activity in an incubation medium after this has been exposed to foreign materials. Samples to be tested were cleaned in $70 \%$ ethanol. Their incubation in a citrated plasma during $15 \mathrm{~min}$ at $37^{\circ} \mathrm{C}$ activated the intrinsic pathway of the coagulation until the formation of factor XIa was completed. Calcium was required for the last part of the coagulation cascade and added phospholipids mimicked phospholipid compounds present at the surface of activated platelets. Thus, all the steps of the cascade that initiated thrombin generation were supposed to be activated. Aliquots were withdrawn at times 0, 1, 2, 4 and 6 min (for weakly reactive samples) after calcium and phospholipid introduction. A chromogenic substrate was then added, which is cleaved by thrombin to yield a yellow-coloured product. Optical densities were read with a Genios Spectra Fluor Plus plate reader (Tecan AG, Hombrechtokon, Switzerland) at $405 \mathrm{~nm}$ with 570 $\mathrm{nm}$ as reference wavelength. Material-generated thrombin activity was calculated by comparison with a calibration curve obtained simultaneously from known thrombin concentrations.

Controls proposed in the kit were LDPE, PDMS and MS plates. MS was considered as highly reactive, PDMS as intermediary reactive and LDPE as weakly reactive. The tested samples had an identical total surface, about $0.6 \mathrm{~cm}^{2}$. The tests have been conducted with two identical samples each in duplicate aliquots.

\section{Platelet adhesion \\ Preparation of human platelet suspension}

Blood from drug-free volunteers was taken into tri-sodium citrate $(3.2 \% \mathrm{w} / \mathrm{v})$ for platelet aggregation experiments. The ratio of whole blood to anticoagulant was 9:1. Whole blood (fractions of $50 \mathrm{ml}$ ) was centrifuged for 15 $\mathrm{min}$ at $200 \mathrm{~g}$. The plasma fraction containing tri-sodium salt was centrifuged at $1000 \mathrm{~g}$ for $30 \mathrm{~min}$. The supernatant was discarded and platelets were then suspended in fresh medium containing $\mathrm{NaCl}(137 \mathrm{mM}), \mathrm{KCl}(1 \mathrm{mM})$, glu- 


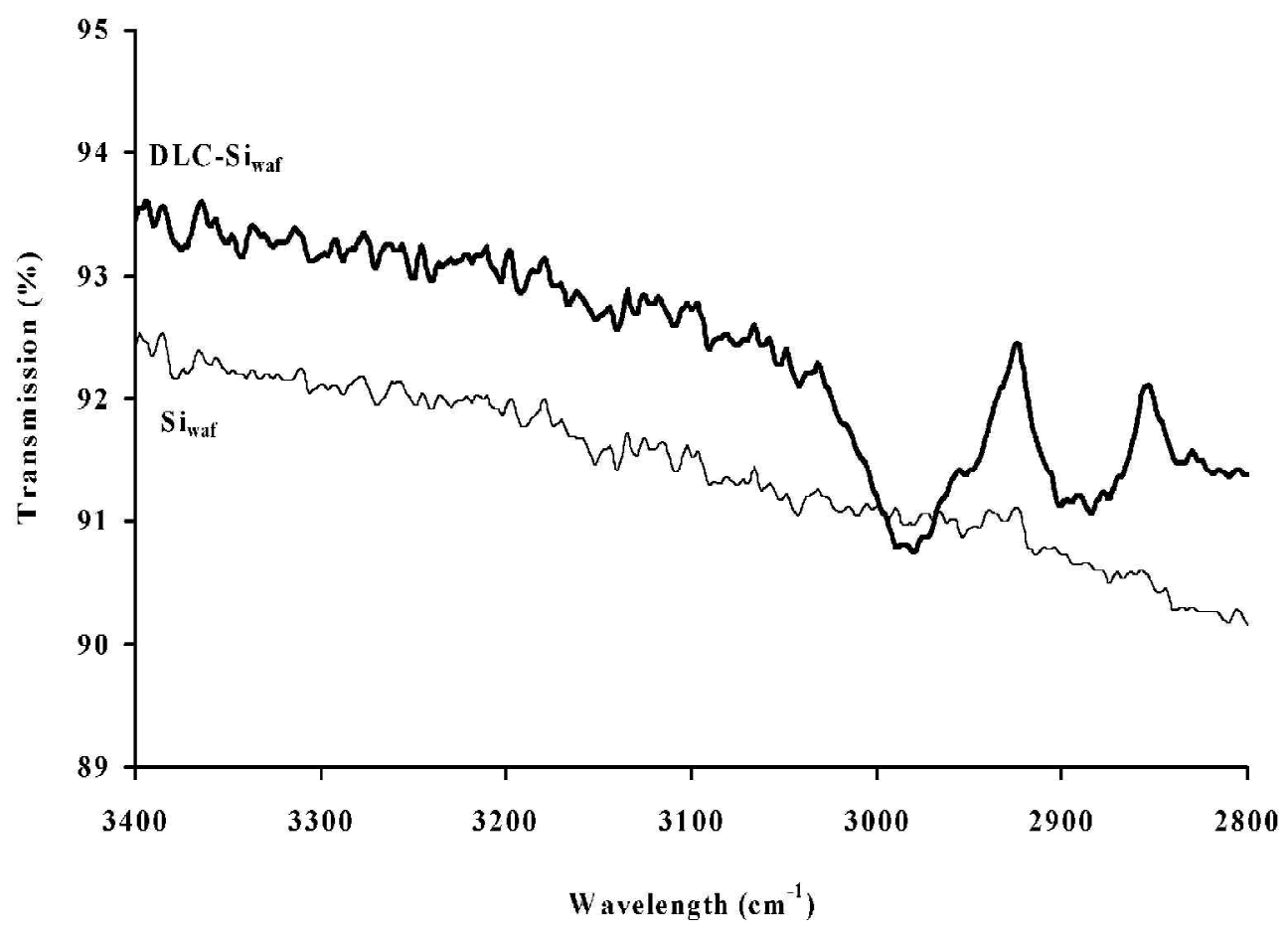

Figure 1. ATR-FTIR spectra of DLC-coated and uncoated $\mathrm{Si}_{\text {waf }}$ in the range $3400-2800 \mathrm{~cm}^{-1}$.

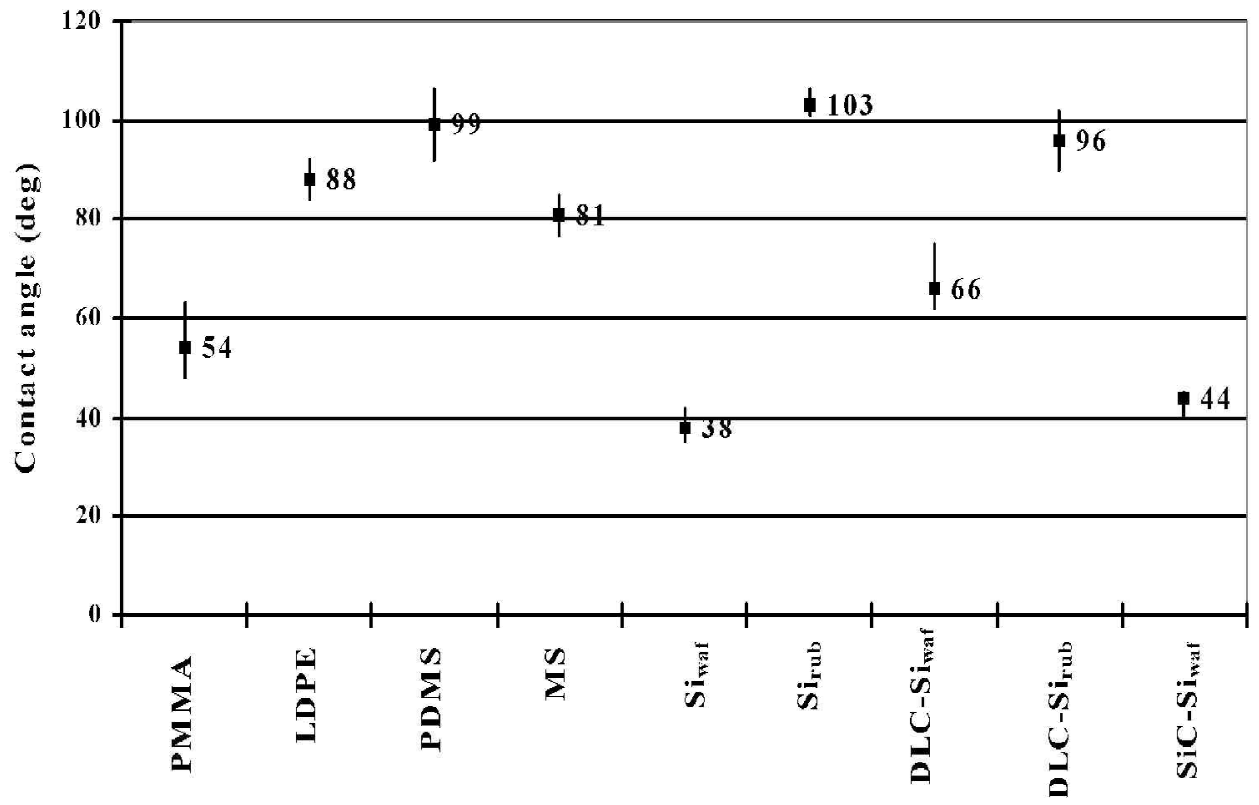

Figure 2. Contact angle measurements between $2 \mu l$-water drop and sample surface in air. 


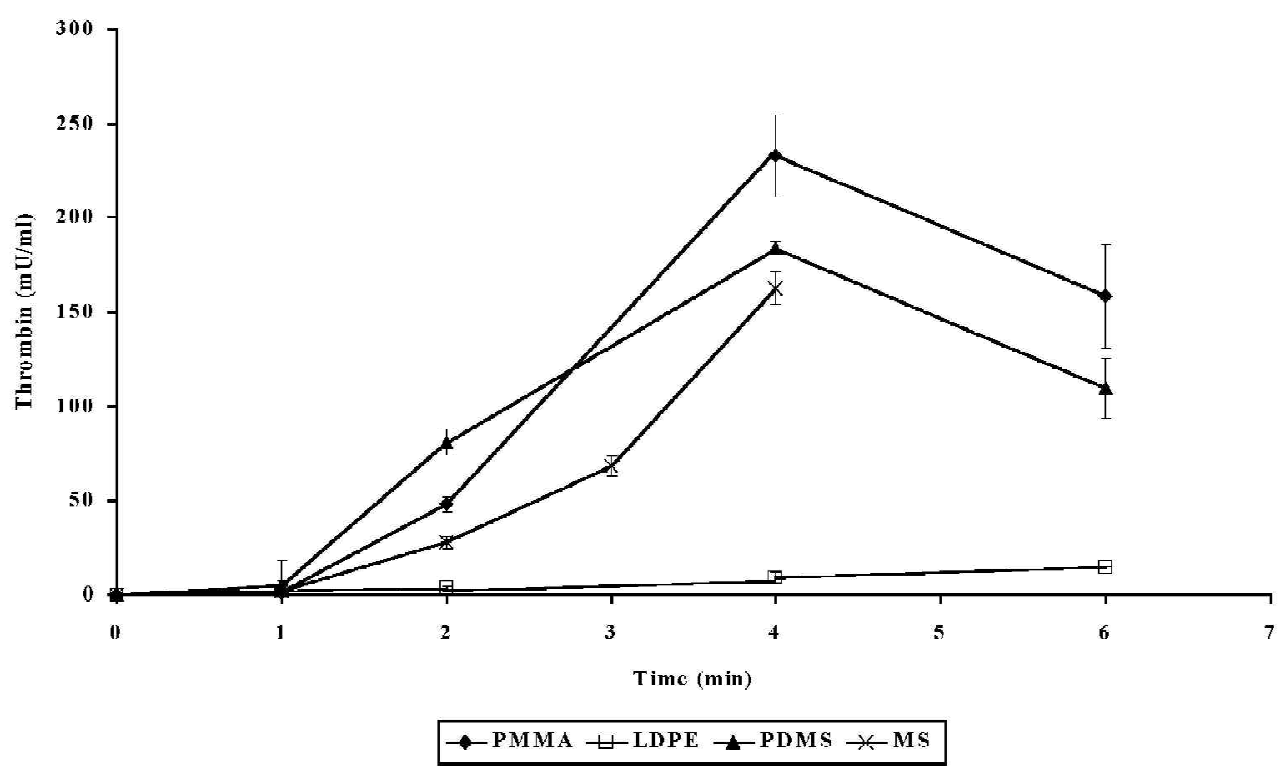

Figure 3. Thrombin generation curves measured for PMMA, LDPE, PDMS and MS.

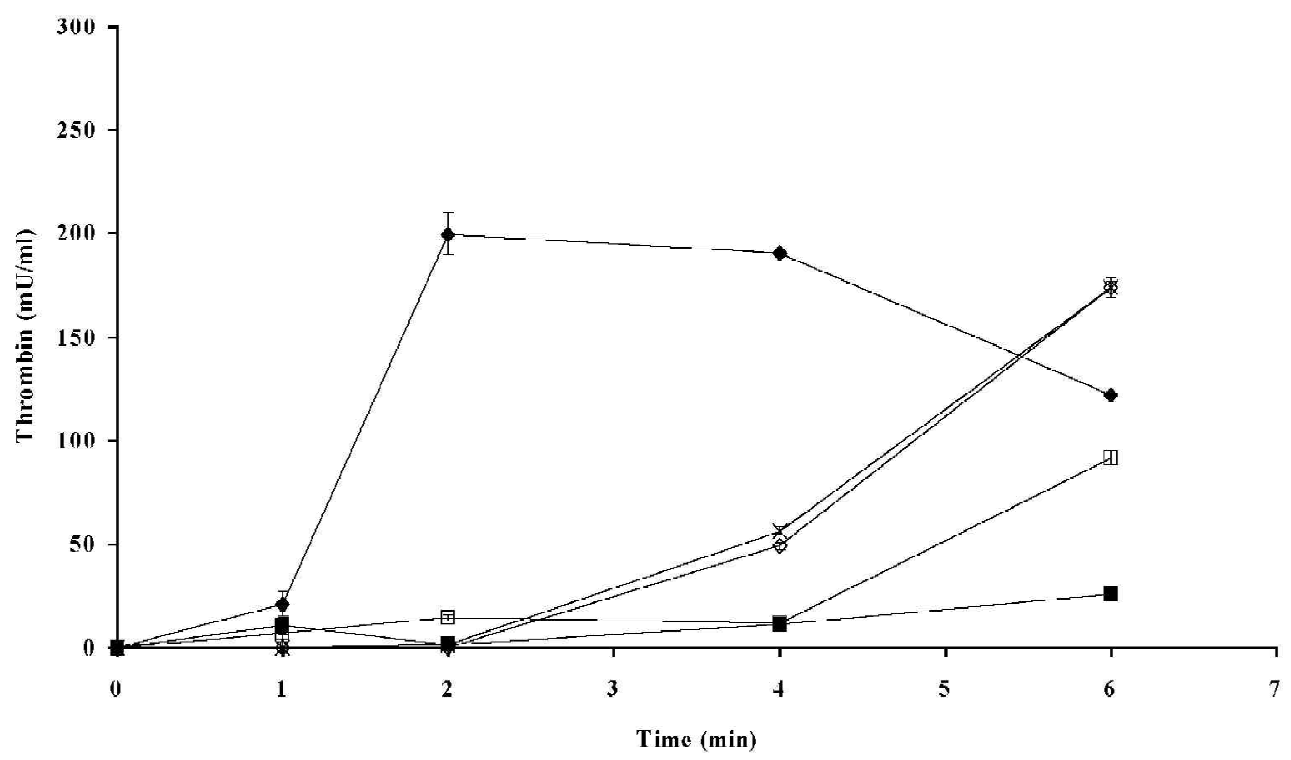

$\multimap-$ Siwaf - -Sirub $\prec$ DLC-Siwaf $\neg$ DLC-Sirub $\nVdash$ SiC-Siwaf

Figure 4. Thrombin generation curves measured for $\mathrm{Si}_{\text {waf }}, \mathrm{Si}_{\text {rub }}$, DLC-Si $\mathrm{waf}_{\text {waf }}, \mathrm{DLC}-\mathrm{Si}_{\mathrm{rub}}, \mathrm{SiC} \mathrm{Si}_{\text {waft }}$

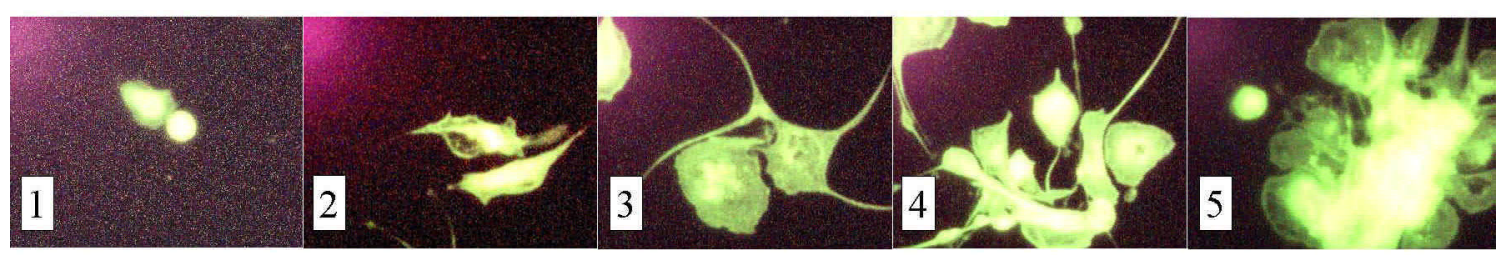

Figure 5. Fluorescence microscopy of various platelet morphologies. $X, Y=25 \mathrm{~mm}, 20 \mathrm{~mm}$. 
Samples

PMMA

LDPE

MS

$\mathrm{Si}_{\text {waf }}$

$\mathrm{Si}_{\text {rub }}$

DLC-Si $i_{\text {waf }}$

DLC-Si ${ }_{\text {rub }}$
SEM imaging
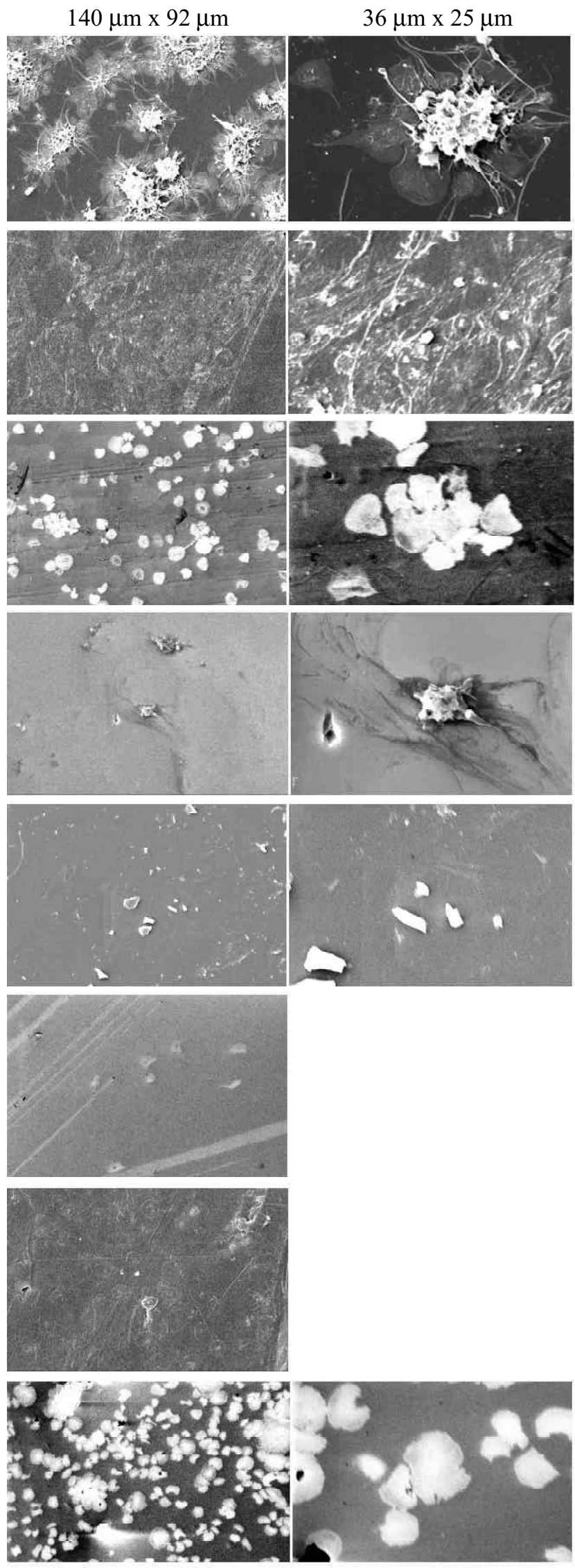

\section{Morphological change}

Dense platelet layer Type 5

Dense formation of pseudopodia

Mainly type 2 and 3

Dense platelet layer Type 5

Low density of platelets Type 3

Few platelets and fragments

Type 1

Few platelets Type 1

Few platelets, filamentous areas

Type 1 and 2

Dense platelet layer Type 5

Figure 6. SEM of adherent platelets on sample surface. Platelet morphological changes are described according to the categorization shown in Figure 5. 
cose $(5.5 \mathrm{mM})$ in Hepes buffer $20 \mathrm{mM}$ pH 7.4 and conserved at $4{ }^{\circ} \mathrm{C}$ during titration of the suspension in a Neubauer haemocytometer. The suspension was then adjusted to a concentration of $2 \times 10^{8}$ platelets $/ \mathrm{ml}$.

\section{Platelet incubation and microscopy imaging}

Two samples of each material (native, reference and treated) were incubated in the previously prepared platelet suspension according to the method developed by Frank et al. (2000). After incubation in platelet suspension, glutaraldehyde was used to fix the platelets on the material surfaces, and imaging was done by epifluorescence and scanning electron microscopy (SEM).

Briefly, samples were immerged in $0.8 \mathrm{ml}$ of platelet suspension and incubated for $30 \mathrm{~min}$ at $37^{\circ} \mathrm{C}$. After washing in PBS, platelets were fixed by glutaraldehyde $(1.5 \%$ solution in PBS) at room temperature. To be observed by SEM (Leo 435VP, Leo Electron Microscopy Ltd, Cambridge, England), samples were dehydrated in ethanol and dried. Polymeric samples were sputtered coated with 10 $\mathrm{nm}$ of gold. For fluorescence observations, after glutaraldehyde fixation, a drop of fluorescent mounting medium (Dako, Hamburg, Germany) was added. The epifluorescence of the labelled platelets was detected by using a digital camera (DN 100, Nikon Co., Tokyo, Japan) mounted on the front port of an inverted microscope (Eclipse TE300, Nikon Co.). A vapour mercury lamp (HBO 103 W/2, 100 W, Osram, Munich, Germany) selectively filtered within the range 450-490 nm was used as excitation light. A long-pass filter (Nikon filter block B2A) allowed the fluorescence signal to be detected for wavelengths longer than $515 \mathrm{~nm}$. The platelet morphology, platelet density and pseudopodium leaching were observed and evaluated. Observations have not been performed on PDMS surfaces because of the sample roughness.

\section{Complement convertase attachment}

The complement convertase assay was provided by HaemoProbe bv as a ready-to-use test kit. Samples were incubated in plasma for $15 \mathrm{~min}$ at room temperature. Complement factors bound to the surface of the materials, and a complement convertase complex formation followed. Samples were washed to eliminate unbound complement proteins. Then, they were incubated during $24 \mathrm{~h}$ at room temperature in a medium containing specific chromogenic substrate having an amino-acid sequence similar to the $\mathrm{C} 5$ cleavage site. Cleavage of the substrate was quantified by measuring the optical density (OD) at $405 \mathrm{~nm}$. Blank was obtained by incubation of a LDPE plate in $0.9 \%$ $\mathrm{NaCl}$ replacing blood plasma. Results were expressed by the values of OD $/ 24 \mathrm{~h} / \mathrm{cm}^{2}$. Controls provided with the kit were LDPE, PDMS and MS plates. MS and PDMS were considered as reactive and LDPE as weakly reactive. The tests have been conducted with two identical samples each in duplicate aliquots.

\section{Results}

\section{ATR-FTIR}

The ATR-FTIR spectrum of the DLC-coated $\mathrm{Si}_{\text {waf }}$ showed a strong adsorption between 2800 to $3400 \mathrm{~cm}^{-1}$ (Figure 1). This region is characteristic for $\mathrm{C}-\mathrm{H}$ stretch vibrations from $\mathrm{sp}^{3}$ - and $\mathrm{sp}^{2}$-hybridized $\mathrm{CH}_{\mathrm{n}}$ and high intensity indicates high hydrogen content. Main peaks were located at 2980, 2945 and $2884 \mathrm{~cm}^{-1}$. According to the literature (Fedosenko et al., 2001; Lung et al., 2001), these peaks could be attributed to the $\mathrm{CH}_{n}$ bonds of $\mathrm{sp}^{3}$-hybridized $\mathrm{CH}_{3}$ (at $2980 \mathrm{~cm}^{-1}$ ), $\mathrm{sp}^{2}$-hybridized and $\mathrm{sp}^{3}$-hybridized $\mathrm{CH}_{2}$ (at $2945 \mathrm{~cm}^{-1}$ ) and $\mathrm{sp}^{3}$-hybridized $\mathrm{CH}_{3}$ (at $2884 \mathrm{~cm}^{-1}$ ). $\mathrm{sp}^{1}$ $\mathrm{CH}$ has not been detected in the range $3200-3400 \mathrm{~cm}^{-1}$. The relative intensity of the $\mathrm{sp}^{3}-\mathrm{CH}_{3}$ groups dominates the contribution to the total adsorption.

Within the given acquisition resolution, we have not any observed differences between spectra of the native $\mathrm{Si}_{\text {waf }}$ and the coated $\mathrm{SiC}-\mathrm{Si}_{\text {waf }}$.

\section{Contact angle measurements}

The results of the contact angle measurements are compiled in Figure 2. From these measurements, three groups of samples can be distinguished: 1- hydrophobic samples : LDPE, PDMS, MS, $\mathrm{Si}_{\text {rub }}, \mathrm{DLC}-\mathrm{Si}_{\text {rub }}, 2$ - intermediary hydrophobic samples : PMMA, DLC-Si ${ }_{\text {waf }}$, and 3- relatively hydrophilic samples : $\mathrm{Si}_{\text {waf }}, \mathrm{SiC}_{-} \mathrm{Si}_{\text {waf }}$. The presence of the DLC coating did not drastically change the wettability of the sample surface.

\section{Thrombin generation}

Accelerated and excessive thrombin generation is the major event leading to the development of haemostatic

Table 1. Thrombin generation.

\begin{tabular}{|l|c|c|c|c|c|c|c|c|c|}
\hline samples & PMMA & LDPE & PDMS & MS & $\mathrm{Si}_{\text {waf }}$ & $\mathrm{Si}_{\text {rub }}$ & DLC-Si $_{\text {waf }}$ & DLC-Si $_{\text {rub }}$ & $\mathrm{SiC} \mathrm{Si}_{\text {waf }}$ \\
\hline $\begin{array}{l}\text { Total amount } \\
(\mathrm{mU} / \mathrm{ml})\end{array}$ & $233 \pm 15$ & $9 \pm 3$ & $183 \pm 3$ & $162 \pm 8$ & $190 \pm 18$ & $11 \pm 10$ & $49 \pm 12$ & $12 \pm 10$ & $56 \pm 5$ \\
\hline $\begin{array}{l}\text { Rate } \\
\left(\Delta \mathrm{mU} / \mathrm{ml} / \mathrm{min} / \mathrm{cm}^{2}\right)\end{array}$ & $7721 \pm 775$ & $226 \pm 130$ & $6266 \pm 2500$ & $6728 \pm 928$ & $14894 \pm 1314$ & $881 \pm 333$ & $5212 \pm 1166$ & $3308 \pm 916$ & $4900 \pm 683$ \\
\hline $\begin{array}{l}\text { Rate range } \\
\left(\Delta \mathrm{mU} / \mathrm{ml} / \mathrm{min} / \mathrm{cm}^{2}\right)\end{array}$ & - & $100-1100$ & $4600-13300$ & $2700-8100$ & - & - & - & - & - \\
\hline
\end{tabular}

Thrombin generation total amount (measured at $4 \mathrm{~min}$ ) and rate (steepest slope of the curve measured between two measured time points). The rate range was given by the kit producer and was dependent of the used lot. 


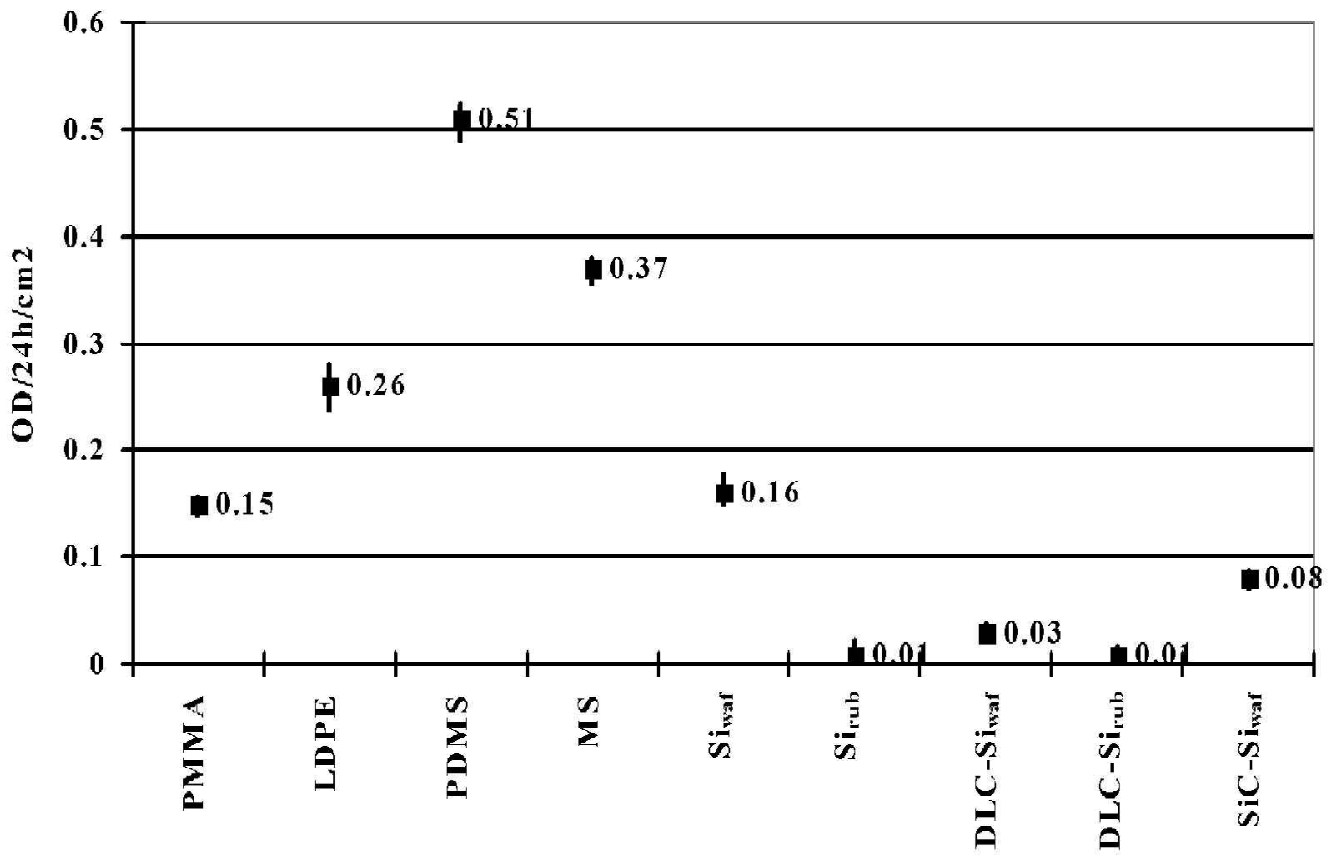

Figure 7. Amount of complement convertase bound to the sample surface.

abnormalities, such as substantial consumption of platelets, fibrinogen, labile coagulation factors and dangerous bleeding. The general shape of a thrombin generation curve shows a short lag time and a fairly quick rise to reach a maximum (Hemker et al., 1996). Figures 3 and 4 show the amount of thrombin generated in citrated plasma by the nine tested samples. In Table 1, some data obtained from the curves are compiled: the total amount of thrombin generated in the plasma at $4 \mathrm{~min}$, and the generation velocity calculated between two points of the steepest positive slope of the curve for each sample.

From the start until $1 \mathrm{~min}$, all levels were within the normal range and no difference between samples was observed. After the first minute, PMMA, PDMS and $\mathrm{Si}_{\text {waf }}$ exhibited a strong increase of thrombin amount until 4 min followed by a slow decrease up to 6 min. MS was mentioned as a surface promoter of thrombin generation and the last measurement on this sample was done at 4 min. LDPE and $\mathrm{Si}_{\text {rub }}$ could be considered as not or only minimally reactive. Their curves were regular with thrombin amounts less than $25 \mathrm{mU} / \mathrm{ml}$. DLC and $\mathrm{SiC}$ coated samples showed a lag time of about $4 \mathrm{~min}$ and a maximal measured value of thrombin at 6 min between 90 and $175 \mathrm{mU} / \mathrm{ml}$. Thrombin generation rates were high for PMMA, PDMS and MS, while the highest rate value (2-3 times higher) was obtained for the $\mathrm{Si}_{\text {waf }}$ sample. The generation rate was slower for the coated DLC-Si ${ }_{\text {waf }}$, DLC$\mathrm{Si}_{\text {rub }}$ and $\mathrm{SiC}-\mathrm{Si}_{\text {waf }}$

\section{Platelet adhesion}

During activation, the platelets attach to the sample surface and they change in shape in developing pseudopodia versus their activation level. Well-defined sequence of morphological changes can be classified as (Barnhart et al., 1972; Ko et al., 1993; Goodman et al., 1989): 1- round or discoid, 2- dendritic or early pseudopodial, without flattening, 3-spread-dendritic or intermediate pseudopodial, 4- spread or late pseudopodial, 5- fully spread (Figure 5). After activation, platelets contract and release the contents of their granules into the extracellular environment. These granular extracts activate other platelets, provoke irreversible platelet aggregation and lead to the formation of a platelet thrombus.

The morphology of the attached platelets after $30 \mathrm{~min}$ of incubation is displayed in Figure 6. Almost all classified morphologies are presented, from isolated circular cells to aggregates with a strong spreading of pseudopodium network. Platelet surface coverage was very prominent on PMMA, MS and $\mathrm{SiC}-\mathrm{Si}_{\text {waf }}$ surfaces with a strong aggregation on PMMA and MS. On these surfaces, the platelets were flat and widely spread. Observed platelets on MS and PMMA seemed to have reached complete cytoskeletal reorganization, with a tendency to achieve complete circumferential and confluent filamentous areas around aggregates. LDPE samples showed a dense formation of pseudopodia with few developed platelets. Platelets adhered to the $\mathrm{Si}_{\text {waf }}$ surface to a lesser extent than to PMMA, MS or $\mathrm{SiC}_{-} \mathrm{Si}_{\text {waf }}$ surfaces, but they were in an activated state with the presence of long pseudopodia. In the cases of $\mathrm{Si}_{\text {rub }}, \mathrm{DLC}-\mathrm{Si}_{\text {waf }}$ and DLC-Si $\mathrm{iub}_{\text {rub }}$, the few adherent platelets remained in discoid form, typically 2 to $5 \mathrm{~mm}$ across. Some filamentous areas were observable on the DLC-Si ${ }_{\text {rub }}$ surface. 
Table 2. Recapitulation of results.

$\begin{array}{lccccccccc} & \text { PMMA } & \text { LDPE } & \text { PDMS } & \text { MS } & \text { SiW } & \text { SiR } & \text { DLC-SiW } & \text { DLC-SiR SiC-SiW } \\ \text { Contact angle } & + & ++ & ++ & ++ & - & ++ & + & ++ & - \\ \text { Thrombin generation } & ++ & - & + & ++ & +++ & - & + & + & + \\ \text { Platelet activity } & ++ & + & \text { NA } & ++ & + & -- & -- & -- & ++ \\ \text { Complement activation } & - & - & ++ & + & - & -- & -- & -- & - \\ \text { Hemocompa-tibility } & - & + & + & - & - & ++ & ++ & ++ & -\end{array}$

Very high $(+++)$, High $(++)$, Moderate $(+)$, Low (-), Inactive (- -).

\section{Complement convertase attachment}

Levels of enzymatic factors, C3 and C5 convertases, adsorbed on the sample surfaces were quantified using the available testing kit. The results are presented in Figure 7 .

All native substrates, except $\mathrm{Si}_{\text {rub }}$, induced an adsorption of complement convertase, with a high level for LDPE, PDMS and MS. The amount of adsorbed convertases was moderate for PMMA and $\mathrm{Si}_{\text {waf }} . \mathrm{Si}_{\text {rub }}$ as well as DLC- and SiC-coated surfaces could be qualified as inactive for complement factor binding.

\section{Discussion}

Blood coagulation and tissue necrosis on foreign devices implanted in or in contact with the body are multifactor processes with dependent tangles of steps. The initial local response to the presence of a foreign surface in the body is mainly catalysed by surface-adsorbed proteins that trigger numerous processes such as cellular activation, inflammation and complement activation, and attraction of circulating platelets (Figure 8).

Generation of thrombin is the critical event of the coagulation process. Thrombin plays a key role in the formation of blood clot through diverse activities. It is known as a procoagulant entity able to generate its own formation in promoting the activity of several clotting factors and the cleavage of fibrinogen to fibrin. It also activates different cells and the platelets, thus leading to arterial thrombosis. However, the function of thrombin is very difficult to evaluate as this enzyme strongly influences many different processes, such as inflammation, coagulation, haemostasis, thrombosis and wound healing. PMMA, PDMS, MS and $\mathrm{Si}_{\text {waf }}$ native surfaces led to the activation of coagulation with a significant increase of thrombin generation after $1 \mathrm{~min}$ in contact with plasma. $\mathrm{Si}_{\text {waf }}$ was the most activating surface. On this substrate, thrombin generation reached a maximum of approximately $200 \mathrm{mU} / \mathrm{ml}$ after $4 \mathrm{~min}$ of incubation in plasma. For the LDPE and $\mathrm{Si}_{\text {rub }}$ samples, thrombin generation was dramatically delayed and reduced with a maximal value at $25 \mathrm{mU} / \mathrm{ml}$ after $6 \mathrm{~min}$ incubation. These samples did not seem to propagate and amplify the blood clotting proc- ess by the thrombin generation event. Thrombin formation is much more retarded in plasma exposed to DLC- or $\mathrm{SiC}$-coated than to native $\mathrm{Si}_{\text {waf }}$ The time of onset of thrombin generation is about 4 min after which an increase to a maximal amount of $174 \mathrm{mU} / \mathrm{ml}$ was observed. In the case of the $\mathrm{Si}_{\text {rub }}$ sample, the DLC coating seemed to stimulate a low thrombin formation in contrast to the native sample. On the other hand, DLC and SiC coatings on $\mathrm{Si}_{\text {waf }}$ delayed the induction of thrombin activity that might delay the propagation of the blood-clotting process, but reached about the same maximum levels.

Although thrombin is one of the most potent agents contributing to platelet activation, which induces thrombosis, the investigation of platelet adhesion must support the thrombogenicity evaluation of biomaterials since clotting factors and activators from platelets strongly contribute to the coagulation process (van Oeveren et al., 2002).

Platelets are anuclear discoid cells that circulate in the blood in a quiescent state with an average size of $3 \mu \mathrm{m}$. In response to an activating stimulus like adsorbed adhesion proteins, platelets become activated and accelerate thrombosis by different ways: significant morphological changes, platelet-platelet aggregation and biochemical actions (secretion contents from alpha and dense cytoplasmic storage granules). Wide flat shapes are strongly associated with activation of granular release mechanisms. Fully spread morphologies were clearly observable for PMMA, MS and SiC- $\mathrm{Si}_{\text {waf }}$, and to a lesser extent, for LDPE and $\mathrm{Si}_{\text {waf }}$ In contrast, $\mathrm{Si}_{\text {rub }}$ and DLC-coated materials demonstrated strongly reduced platelet adhesion and activation.

Synthetic surfaces do not only activate the coagulation cascade but also provoke inflammatory responses involving the complement system. Some complement factors act as mediators of inflammation and they are recognized by specific receptors on polymorphonuclear leukocytes, monocytes, and other cells (Janatova, 1992). The host inflammatory immune response to a foreign body is provoked by leukocytes after recognition and adhesion via ligand receptor superfamilies to surface-adsorbed proteins. These receptors are promoted by the activation of the complement cascade (Ziats et al., 1988; Kao, 2000). 


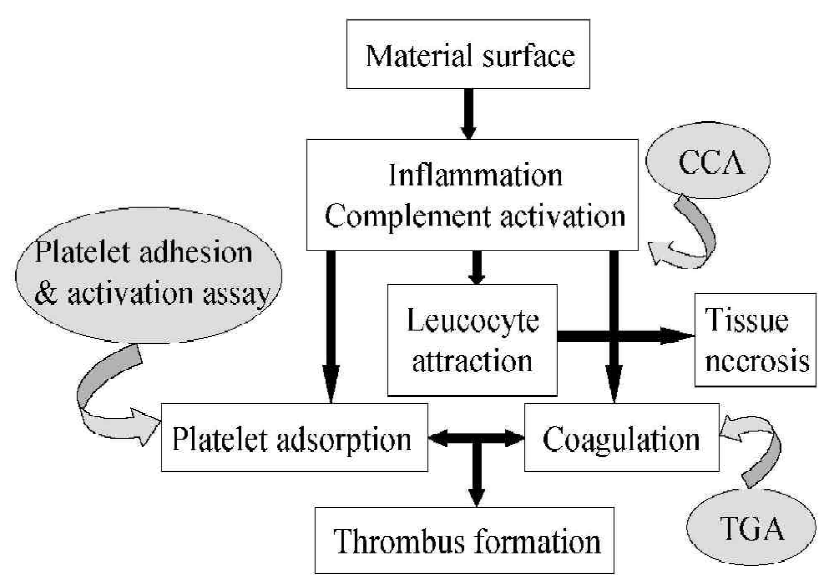

Figure 8. Schematic representation of thrombogenic events on a body foreign surface. Tests used in this paper are indicated.

All the processes activating the complement cascade (classical, lectin and alternative pathways) (Volanakis et al., 1998) converge in the assembly of C 3 and C5 convertases. PDMS, MS, PMMA, LDPE, $\mathrm{Si}_{\text {waf }}$ and $\mathrm{SiC}-\mathrm{Si}_{\text {waf }}$ all provoked a complement convertase adsorption whereas DLCcoated surfaces and $\mathrm{Si}_{\text {rub }}$ did not induce any complement activity.

Thrombin generation, platelet adhesion and complement factor activation are three steps extracted from the blood coagulation cascade and the inflammatory events. They allow an overview of the coagulation induction, the thrombotic ability from platelet adhesion and activation, and the inflammatory response. They constitute a representative panel for an in vitro evaluation of the thrombogenicity (Figure 8).

In this regard, results were schematically compiled in Table 2 and the in vitro blood contacting behaviour for each sample was deduced. The following classification, from the most to the less haemocompatible surface can be deduced: $\mathrm{Si}_{\text {rub }} / \mathrm{DLC}-\mathrm{Si}_{\text {rub }} / \mathrm{DLC}-\mathrm{Si}_{\text {wat }} / \mathrm{LDPE} / \mathrm{PDMS} / \mathrm{SiC}$ $\mathrm{Si}_{\text {wat }} / \mathrm{Si}_{\text {waf }} / \mathrm{PMMA} / \mathrm{MS}$. LDPE, PDMS, SiC-Si $i_{\text {waf }}, \mathrm{Si}_{\text {waf }}$, PMMA and MS could be considered as having a low haemocompatibility, and tend to be thrombogenic and to induce inflammation in vitro. It means that it is expected that their in vivo use could lead to blood clotting at the site of implanted biomaterial, continual shedding of thrombi and depletion of platelets from the blood stream. DLC-Si $i_{\text {waf }}$ and DLC-Si $i_{\text {rub }}$ and $\mathrm{Si}_{\text {rub }}$ showed a delayed clotting time, less adherent platelets, no aggregation, less pseudopodia of the adherent platelets and no activated complement factor adsorption. These samples represent potential candidates for further in vivo blood-contacting experiments. This is in accordance with the work of Linder et al. (2002) that reported the good biocompatibility of a DLC-coated surface in presence of blood monocytes in vitro. Eriksson and Nygren (1996) compared the behaviour of carbon and gold, and found that graphite was more thrombogenic than gold but less inflammatory. The smoothness and inert nature of this type of surfaces could explain their good haemocompatibility.

Some authors have correlated the blood-contacting behaviour of surfaces with their interfacial free energy and surface chemistry (Goodman et al., 1989, Hunt et al., 1997). They demonstrated a relationship between surface hydrophobicity and the in vitro activation of coagulation, fibrinolysis and platelet deposition. The most hydrophobic surfaces appeared to cause least activation of coagulation but most activation of platelets. In our study, a direct correlation between contact angle value and the biological test results cannot be suggested from Table 2 .

By superrimposing Figures 2 and 7, we observe (i) a matching between the location of the points for PMMA, LDPE, PDMS, MS and $\mathrm{Si}_{\text {waf }}$ while (ii) no such matching can be found for DLC-and SiC-coated materials. Furthermore, the former samples triggered platelet activation and, except LDPE, thrombin generation. It may thus be hypothesized that interfacial surface free energy is related to the in vitro haemocompatibility behaviour, for these samples in absence of DLC-coating. In the case of DLCcoated samples, the more chemically inert nature and the smoothness of the coating seem to be the predominant factors that govern the response of the blood proteins to the surface. However, this hypothesis is not applicable to the $\mathrm{SiC}-\mathrm{Si}_{\text {waf }}$ surface.

\section{Conclusion}

It has been shown that a DLC coating prolonged the clotting time and tended to suppress the platelet and complement convertase activation, in contrast to PMMA, LDPE, PDMS, MS and $\mathrm{Si}_{\text {waf }}$ native substrates. The inert nature and the smoothness of the DLC-coated surface appeared to dominantly explain the good in vitro haemocompatibility. The acceptability of results of thrombin generation, platelet adhesion and complement convertase in vitro assays address the samples to further evaluation for blood contacting applications, mainly in vivo experiments. $\mathrm{SiC}$ coating has been considered as thrombogenic. The electronic character of the deposited semi-conductive film is supposed to influence the electron transfer between the implant surface and proteins and cells playing a key role in thrombosis generation. The relationship between the electronic properties of the coated material and their haemocompatibility will be exposed in a forthcoming paper.

\section{Acknowledgements}

This work is supported by the COST Action 527 (Contract $n^{\circ}$ BBW/OFES-NBC01.0051) from the Swiss government, which is acknowledged.

\section{References}

Barnhart MI, Walsh RI, Robinson JA (1972) A threedimensional view of platelet responses to chemical stimuli. Ann N Y Acad Sci 201: 360-390.

Bohler J, Kramer P, Gotze O, Shartz P, Scheler F (1983) Leukocyte counts and complement activation during pump-driven and arteriovenous haemofiltration. Cont Hephrol 36: 15-17. 
Chenoweth DE (1984) Complement activation during hemodialysis: clinical observations, proposed mechanisms and theoretical implications. Artif Organs 8: 2817.

Chenoweth DE (1986) Complement activation produced by biomaterials. Trans Am Soc Artif Intern Organs 32: 228-232.

Craddock PR, Fehr J, Dalmasso AP, Brighan KL, Jacob HS (1977) Hemodialysis leucopenia; Pulmonary vascular leukostasis resulting from complement activation by dialyzer cellophane membranes. J. Clin Invest 59: 879-888.

Dawie EW, Ratnoff OD (1964) Waterfall sequence for intrinsic blood clotting. Science 145: 1310-1312.

De Scheerder I, Szilard M, Yanming H, Ping XB, Verbeken E, Neerinck D, Demeyere E, Coppens W, van der Werf F (2000) Evaluation of the biocompatibility of two new diamond-like stent coatings (Dylyn) in a porcine coronary stent model. J Invasive Cardiol 12: 389394.

Eriksson C, Nygren H (1996) The initial reactions of graphite and gold with blood. J Biomed Mater Res 37: 130-136.

Fedosenko G, Korsec D, Schwabedissen A, Engemann J, Braca E, Kenny JM (2001) Comparison of diamondlike carbon films synthesized by $2.45 \mathrm{GHz}$ microwave and 13.56 MHz multi-jet radiofrequency plasma sources. Diamond and Related Materials 10:920-926.

Ferry JD, Morrison PR (1947) Preparation and properties of serum and plasma proteins. VIII. The conversion of human fibrinogen to fibrin under various conditions. J Am Chem Soc 69: 388-399.

Frank RD, Dresbach H, Thelen H, Sieberth HG (2000) Glutardialdehyde induced fluorescence technique (GIFT): A new method for the imaging of platelet adhesion on biomaterials. J Biomed Mater Res 52: 374-381.

George JN (2000) Platelets. Lancet 355: 1531-1539.

Goodman SL, Grasel TG, Cooper SL, Albrecht RM (1989) Platelet shape change and cytoskeletal reorganization on polyurethaneureas. J Biomed Mater Res 23: 105 123.

Gutensohn K, Beythien C, Bau J, Fenner T, Grewe P, Koester R, Padmanaban K, Kuehnl P (2000) In vitro analyses of diamond-like carbon coated stents : Reduction of metal ion release, platelet activation and thrombogenicity. Thromb Res 99: 577-585.

Hemker HC, Willems GM, Béguin S (1996) A computer assisted method to obtain the prothrombin activation velocity in whole plasma independent of thrombin decay processes. Thromb Haemost 56: 9-17.

Hunt BJ, Paratt R, Cable M, Finch D, Yacoub M (1997) Activation of coagulation and platelets is affected by the hydrophobicity of artificial surfaces. Blood Coagul Fibrinolysis 8: $223-231$.

Janatova J (1992) Activation and control of complement, inflammation and infection associated with the use of biomedical polymers. ASAIO J 46: S53-S62.

Jones MI, McColl IR, Grant DM, Parker KG, Parker TL (1999) Haemocompatibility of DLC and TiC-TiN interlayers on titanium. Diamond and Related Materials 52: 457-462.
Jones MI, McColl IR, Grant DM, Parker KG, Parker TL (2000) Protein adsorption and platelet attachment and activation on TiN, TiC and DLC coatings on titanium for cardiovascular applications. J Biomed Mater Res 52: 413421.

Kao WJ (2000) Evaluation of leukocyte adhesion on polyurethanes : the effect of shear stress and blood proteins. Biomaterials 21: 2295-2303.

Ko TM, Lin JC, Cooper SL (1993) Surface characterization and platelet adhesion studies of plasma-sulphonated polyethylene. Biomaterials 14: 657-664.

Lamba NMK, Cooper SL (2001) Interactions of blood with artificial surfaces. In: Colman RW, Hirsh J, Marder VJ, Clowes AW, George JN, eds. Hemostasis and thrombosis: basic principles and clinical practice - Fourth edition. Philadelphia. Lippincott Williams \& Wilkins. pp. 661-672.

Linder S, Pinkowski W, Aepfelbacher M (2002) Adhesion, cytoskeletal architecture and activation status of primary human macrophages on a diamond-like carbon coated surface. Biomaterials 23: 767-773.

Lung BH, Chiang MJ, Hon MH (2001) Growth characterization and properties of diamond-like carbon films by electron resonance chemical vapor deposition. Thin Solid Films 392: 16-21.

MacFarlane RG (1964) An enzyme cascade in the blood clotting mechanism and its function as a biochemical amplifier. Nature 202: 498-499.

Overduin M, de Beer T (2000). The plot thickens: How thrombin modulates blood clotting. Nature Struct Biol 7: 267-269.

van Oeveren W, Haan J, Lagerman P, Schoen P (2002) Comparison of coagulation activity tests in vitro for selected biomaterials. Artif Organs 26: 506-511.

Volanakis JE (1998) Complement enzymes. In: The Human Complement System in Health and Disease. Volanakis JE, Frank MM, eds. Marcel Dekker, New York. pp. 9-32.

Wang X, Zhang F, Li C, Zheng Z, Wang Xi, Liu X, Chen A, Jiang Z (2002) Improvement of blood compatibility of artificial heart valves via titanium oxide film coated on low temperature isotropic carbon. Surf Coat Technol 36: 128-129.

Young BR, Lambrecht LK, Cooper SL (1982) Plasma proteins: Their role in initiating platelet and fibrin deposition on biomaterials. In: Biomaterials: Interfacial Phenomena and Applications. Cooper SL, Peppas NA, eds. Advances in Chemistry Series 199: American Chemical Society, Washington DC. pp. 317-350.

Ziats NP, Miller KM, Anderson JM (1988) In vivo and in vitro interaction of cells with biomaterials. Biomaterials 9: 5-13.

\section{Discussion with Reviewers}

R.Barbucci: Why were morphological changes classified by Fluorescence Microscopy and then analyzed by SEM? Authors: In fluorescence microscopy a fluorochrome is excited with ultraviolet light and the resulting visible fluorescence is viewed. This produces a bright image in a 
dark background. Main advantages of the fluorescence microscopy are the speed, and facility of the imaging, with an easy method for sample preparation. A drop of fluorescent medium has been deposited on the samples after platelet fixation with glutaraldehyde. However, light microscopy exhibits a limit of resolution, which is generally defined as $0.61 \lambda / \mathrm{NA}$, where NA (numerical aperture) is a property of the objective lens determined by its magnification, diameter and refractive index. Typical ranges for the NA are 0.25-1.32. Visible light has an average wavelength of approximately $0.5 \mu \mathrm{m}$ making the maximum limit of resolution approximately $0.2 \mu \mathrm{m}$. The magnitude of the imaging depends on the objectives coupled to the apparatus. Some polymer substrates, as polyurethane, are also coloured by the fluorescent medium and platelets cannot be thus distinguished from the substrates. Furthermore, considering our equipment, samples need to be transparent to be observed. Objectives are placed under the sample and the light comes below. For all these reasons, we are only used this technique to classify platelet morphological changes observed after adhesion on glass, these qualitative criterion of adherent platelet shape changes being very well known and referenced. Measurements could not be done with opaque samples such as silicon wafer and medical steel.

The electron microscope provides additional resolution that allows for the examination of sub-cellular structures and even molecules. The resolution of our SEM is approximately $10 \mathrm{~nm}$ with an effective magnification of up to 20,000 times. Only surface features can be examined (with our SEM). The sample preparation for this imaging technique is much more difficult and time-consuming. It needs the dehydration of fixed platelets, following by a gold coating. All samples can be observed, opaque or transparent, conductor or not (with an additional gold coating). Zooming and enhancement of the magnitude is easy and continuous.

C. Bacquey: Complement activation is investigated through the assay of $\mathrm{C} 3 \mathrm{a}$, and sometimes $\mathrm{C} 5 \mathrm{a}$, the risk being to not take into account the $\mathrm{C} 3 \mathrm{a}$ (C5a) that may adsorb onto the material under test. Assaying convertase may encounter the same type of risk. What are the consequences of having a part of the convertase bound to the material and the other in solution?

Authors: The complement system destroys invading foreign cells and organisms. Its activation results in deposition of fragmented forms $\mathrm{C} 3 \mathrm{~b}$ and $\mathrm{iC} 3 \mathrm{~b}$ on the biomaterial surface, and these $\mathrm{C} 3$ fragments act as ligands for complement receptors on leukocytes. A series of events results in the formation of bound $\mathrm{C} 3 \mathrm{~b}$ and $\mathrm{C} 5 \mathrm{~b}$ and the generation of soluble $\mathrm{C} 3 \mathrm{a}$ and $\mathrm{C} 5 \mathrm{a}$. The soluble compound leads to the chemotaxis, cytokine release, generation of prostaglandins and leukotrienes. Furthermore, sC5b-9 induces platelet activation and expression of P-selectin, which mediates the binding of platelets to leukocytes (Johnson, 1991). As unbound complement proteins were washed, the CCA test used in this study takes into account only the forms bound to the biomaterial surfaces. Bound $\mathrm{C} 3$ and $\mathrm{C} 5$ convertases are representative of the early step on the inflammatory reaction and can be considered as a direct approach of the total amount of complement activation.

C. Bacquey: Authors should have discussed the limits of their study as far as platelets activation is concerned. Platelet may or may not be activated when they get in contact with a surface, but the result is strongly dependant of environmental factors such as the wall shear rate and henceforth the surface morphology. Thus any experimental model which does not take into account the dynamics of a real situation will not allow a correct assessment of the behaviour of platelets; the model used by the authors may allow a comparison of different materials, but they must know that the hierarchy could be different using dynamic conditions, especially if the materials surfaces are not smooth enough.

Authors: Of course our results are limited as our experiments of platelet adhesion take place within in vitro static conditions. A lot of factors cannot be considered, as such the roughness on the sample that is susceptible to create platelet aggregations, surface free energy of the surface that makes vary the local shear flow of platelets. For example, the strength of platelet adhesion can not be determined, although well-spread platelets adhere on a polyurethane in static conditions, platelet deposition was consistently much lower on this polyurethane than on the other materials examined (Goodman et al., 1989; text reference). The role of shear in material-induced cellular activation has been well referenced by Sefton et al. (2000). In most of Sefton's studies, it appears that shear rates influence platelet and leukocyte behaviour. Differences are distinguished during platelet aggregation, platelet adhesion to fibrinogen and von Willebrand factor.

C. Bacquey: Non-adherence of platelets may be misinterpreted. In the early seventies, Gott, on one side, and Kusserow, on the other side, showed that silicon elastomer rings inserted in different vascular sites were not so much loaded with platelets in comparison with other materials. However, Kusserow demonstrated very elegantly that platelets were nevertheless activated; when the silicon elastomer rings were inserted in the renal artery, they kept open but pathological studies of the kidney showed that a lot of capillaries were embolized by platelets aggregates. Authors: The remark of the reviewer is justified. The number of platelets is not directly related to the rate of systemic compounds release. It depends on the stage of platelet activation, which proceeds stepwise; the stimulation by the polymer is transmitted to platelets (Ito et al., 1989). Some of the released substances can provoke localized or systemic reactions such as embolization. In vivo, after surface-mediated aggregation, emboli can detach and disseminate leading to complication. In this study, we observe adhesion and/or aggregation after short-time exposure, which gives indication on the surface properties. The fact that platelet adhesion and spreading, in these conditions appear lower onto the modified surface is a positive results. However, we still need to evaluate the behaviour of the modified material in dynamic condition 
and/or in vivo to clearly document this point as suggested by the reviewer.

L. Koole: What is the exact philosophy behind the choice of diamond-like coating? Of course, the coating is smooth and hard, and this will make attachment of proteins and platelets not so easy. But the coating is not at all biomimetic. It does not resemble the endothelial surface that is found on the healthy blood vessel's lumenal wall. Is the reason for this choice, perhaps, that the technology for applying this coating is already there? Should this be our approach to improved biocompatibility? Or should biomimicry be preferred?

Authors: As mentioned by numerous works, DLC and $\mathrm{SiC}$ coatings show relative good biocompatibility, but could not be deposited onto polymer surface with sufficient affinity, up to now. The challenge of our technological development is to deposit them on soft polymers, especially elastomers. From our point of view, the deposi- tion of these coatings can easily and rapidly lead to improve the biocompatibility of polymeric materials. Of course, this treatment cannot be compared to biomimetic grafted systems which need much more investigations. We believe that the solution of DLC deposition is not so optimal as the biomimetic concept would be, but better than existing products.

\section{Additional References}

Ito Y, Sisido M, Imanishi Y (1989) Platelet adhesion onto protein-coated and uncoated polyetherurethaneurea having tertiary amino groups in the substituents and its derivatives. J Biomed Mater Res 23: 191-196.

Johnson RJ (1991) Complement activation by biomaterials. Prog Clin Biol Res 337: 507-512.

Sefton MV, Cynthia HG, Gorbet MB (2000) What really is blood compatibility? J Biomater Sci Polym Edn 11: $1165-1182$. 